\title{
Nuclear Polarization of Hydrogen Molecules from Recombination of Polarized Atoms
}

\author{
T. Wise, ${ }^{*}$ W. Haeberli, B. Lorentz, ${ }^{\dagger}$ P. A. Quin, F. Rathmann, ${ }^{\dagger}$ B. Schwartz, and T. G. Walker \\ University of Wisconsin-Madison, Madison, Wisconsin 53706
}

A. Wellinghausen, J. T. Balewski, J. Doskow, H. O. Meyer, R. E. Pollock, B. v. Przewoski, and T. Rinckel

Indiana University Cyclotron Facility, Bloomington, Indiana 47405

\author{
Swapan K. Saha \\ Department of Physics and Astronomy, University of Pittsburgh, Pittsburgh, Pennsylvania 15260
}

P. V. Pancella

Department of Physics, Western Michigan University, Kalamazoo, Michigan 49008

(Received 2 February 2001; published 3 July 2001)

\begin{abstract}
The nuclear polarization of $\mathrm{H}_{2}$ molecules formed by recombination of polarized $\mathrm{H}$ atoms on a $\mathrm{Cu}$ surface was measured as a function of external magnetic field and of temperature of the surface. The proton polarization of the molecules was determined by scattering of a longitudinally polarized 203-MeV proton beam in the Indiana University Cyclotron Facility storage ring. The nuclear polarization of the molecules, relative to the polarization of the atoms before recombination, increased from near zero in a weak magnetic field to $0.42 \pm 0.02$ in a $0.66 \mathrm{~T}$ field. A simple model of the relaxation accounts quantitatively for the observations.
\end{abstract}

DOI: $10.1103 /$ PhysRevLett.87.042701

PACS numbers: 29.25.Pj, 34.90. $+\mathrm{q}$

Conventional spin-physics experiments in high energy and nuclear physics use as targets hydrogenous substances such as $\mathrm{NH}_{3}$ at low temperatures, whose protons are polarized by microwave irradiation. However, during the past few years, increased use has been made of polarized hydrogen and deuterium gas targets, which are placed in the circulating beam of storage rings. In order to increase the target thickness over that obtained by a jet of polarized $\mathrm{H}$ atoms, the beam from atomic beam sources is directed into an open cell ("storage cell") in which the atoms make several hundred collisions before escaping from the target [1]. One example is the series of experiments by the HERMES Collaboration, which has studied, e.g., deep inelastic scattering of $27.6 \mathrm{GeV}$ electrons from a polarized $\mathrm{H}$ target at DESY [2]. Other applications are measurements of spin correlation parameters in proton-proton scattering at the Indiana University Cyclotron Facility (IUCF) [3], and studies of electronuclear form factors at Novosibirsk [4] and at the electron storage ring in Amsterdam, NIKHEF [5]. The polarization of the $\mathrm{H}$ atoms in such a target can be monitored by an atomic polarimeter which determines the relative populations of the four hyperfine states of $\mathrm{H}$. However, a fraction of the atoms recombines to form $\mathrm{H}_{2}$, whose nuclear polarization is unknown, which causes a significant systematic error in some of the above measurements.

This raises the interesting question: To what extent is the nuclear polarization of the atoms preserved after recombination? A recent measurement with deuterium [6] reported that the molecules retain the tensor polarization of the atoms, but the statistical uncertainty of the measurements (fraction polarization retained
$=0.81 \pm 0.31)$ was very large. Here we report the first measurements on the recombination of $\mathrm{H}$ atoms and the first study of the dependence on magnetic field and temperature of the surface where recombination takes place.

The idea of the experiment is to compare the nuclear polarization $P_{\text {at }}$ of the atoms from the atomic beam source [7] to the nuclear polarization $P_{\mathrm{mol}}$ of the molecules when the atoms are allowed to recombine on a copper surface. The nuclear polarization is measured by passing a $203 \mathrm{MeV}$ polarized proton beam through a target cell containing the gas to be analyzed, making use of the large spin correlation coefficient $A_{z z}$ in $p p$ scattering, as described in more detail below.

A simplified scale drawing of the equipment is shown in Fig. 1. A beam of polarized $\mathrm{H}$ atoms in a pure spin state (state $1, m_{I}=1 / 2, m_{j}=1 / 2$, see Ref. [8]) passes through a $13 \mathrm{~cm}$ long, tapered entrance tube and enters the recombination cell. In the recombiner, which is made of copper and is lined with copper mesh, the atoms encounter enough wall collisions to lead to almost complete recombination. The $\mathrm{H}_{2}$ molecules (and some remaining atoms) diffuse out of the recombiner into a thin-walled $\mathrm{Al}$ target cell (length $25 \mathrm{~cm}$, diameter $1.2 \mathrm{~cm}$ ) through which the proton beam passes. The recombination cell is separated from the target cell by a remotely operated valve. To measure $P_{\text {at }}$ the valve is closed, in which case the atoms from the polarized beam source diffuse into the target cell with negligible loss in polarization, because they encounter only the Teflon-coated surfaces of the recombiner valve and the target and entrance tubes, which are known $[9,10]$ to strongly inhibit depolarization and recombination. The 


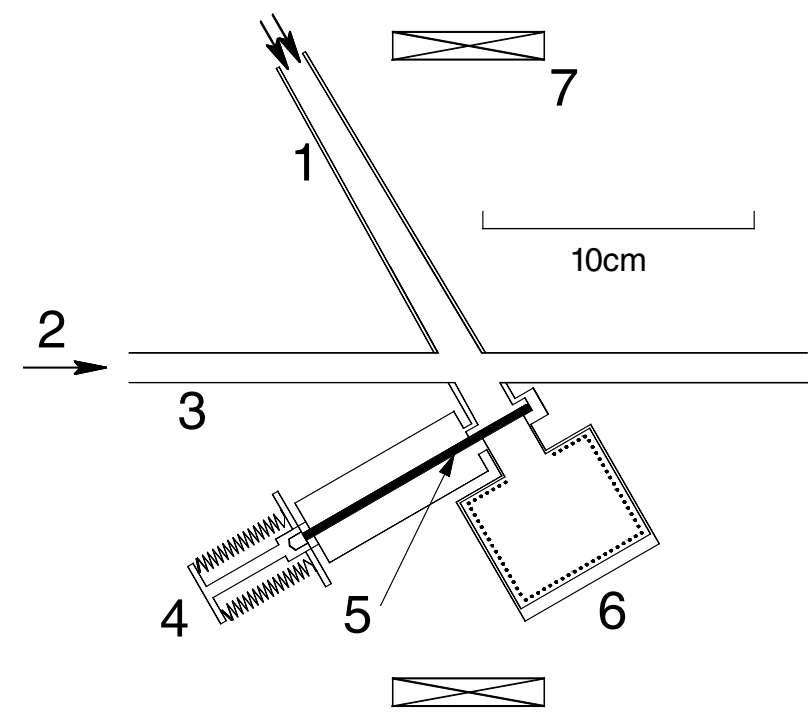

FIG. 1. Schematic scale drawing of recombiner volume and target cell. (1) Tapered Teflon-coated entrance tube for polarized $\mathrm{H}$ atoms; (2) $203 \mathrm{MeV}$ proton beam; (3) Teflon-coated Al target cell; (4) pneumatic valve actuator; (5) Teflon valve; (6) copper recombiner; (7) superconducting current loop.

present measurements determine the polarization $P_{\mathrm{mol}}$ of the $\mathrm{H}_{2}$ molecules, relative to the polarization of the atoms before recombination:

$$
R=P_{\mathrm{mol}} / P_{\mathrm{at}} .
$$

The magnetic field is provided by a superconducting coil (Fig. 1) which produces fields up to $0.66 \mathrm{~T}$ at the recombiner. Thus, the available field is large compared to the critical field of the hyperfine interaction in $\mathrm{H}$ of $50.7 \mathrm{mT}$ [8]. To avoid the large distortion of the proton closed orbit that a transverse $B$ field would have entailed, $B$ was chosen longitudinal ( $z$ direction). Forward wire chambers and scintillators detected protons from $p p$ elastic scattering at lab angles in the range $30^{\circ}-60^{\circ}$. Event identification depended on detection of two protons in coincidence, with the requirement that they be coplanar.

Parity conservation requires the longitudinal analyzing power to be zero. Consequently, to measure the longitudinal target polarization, one needs to resort to a spin correlation measurement (polarized beam and polarized target).

The number of observed $p p$ coincidences for longitudinal beam polarization $p$ and target polarization $P$ can be written as

$$
Y=k t(\alpha)\left[1+p P A_{z z}(\theta)\right]
$$

where the dependence of $Y$ on proton beam current was removed by dividing the observed number of counts by the integrated beam current obtained from a current transformer. In the above equation, target polarization $P$ stands for either $P_{\text {open }}$ or $P_{\text {closed }}$, depending on whether the recombiner valve is open or closed, and the constant $k$ contains factors such as the $p p$ cross section and detector efficiencies. The spin correlation coefficient $A_{z z}$ has recently been shown [11] to be near 1 in the vicinity of $\theta_{\text {lab }}=45^{\circ}$, but the precise values of $p$ and $A_{z z}$ are unimportant here because they cancel in the ratio $P_{\text {open }} / P_{\text {closed }}$. The target thickness $t(\alpha)$ is a function of the degree of dissociation of the target gas because the conductance depends on the mass of the target particles.

Data acquisition cycles were 4 min long. During each cycle, the recombiner valve was switched between the open and the closed position twice. At the end of a cycle, the stored proton beam was dumped. For every third cycle, the polarization of the injected beam was turned off. This allowed taking the ratio of $Y$ [Eq. (2)] for polarized and unpolarized beams to determine $p P_{\text {open }} A_{z z}(\theta)$ or $p P_{\text {closed }} A_{z z}(\theta)$, depending on whether the valve-open or the valve-closed part of the cycle is evaluated. As a check, the beam polarization $p$ was reversed in sign after every polarized cycle.

Ideally, the degree of dissociation, $\alpha$, defined as the fraction of hydrogen which is in atomic form,

$$
\alpha=n_{\mathrm{H}} /\left(n_{\mathrm{H}}+2 n_{\mathrm{H} 2}\right) \text {, }
$$

should be $\alpha_{\text {closed }}=1$ when the recombiner valve is closed, and $\alpha_{\text {open }}=0$ when the valve is open. Under this condition, $R=P_{\mathrm{mol}} / P_{\text {at }}=P_{\text {open }} / P_{\text {closed }}$. Prior to installation in the IUCF storage ring, the recombiner was tested by analyzing the gas emerging from the target tube with a quadrupole mass analyzer. Recombination $>85 \%$ was achieved, but only after lining the inside of the recombiner with copper mesh to increase the number of wall collisions. To guard against changes in the surface properties of the recombiner, the degree of recombination was monitored throughout the measurements, and the deviation from the ideal case was taken into account in the data analysis, as described below.

To monitor $\alpha$, we made use of the yields [Eq. (2)] measured with an unpolarized beam, $Y=k t(\alpha)$. In the ideal case, $Y$ would increase by a factor $\sqrt{2}$ when the recombiner valve is opened, because the gas conductance is proportional to $1 / \sqrt{\mathrm{m}}$, where $m$ is the mass of the particles in the target. In practice, the increase will be $<\sqrt{2}$. The value of $Y_{\text {open }} / Y_{\text {closed }}$ found here is $1.364 \pm 0.004$, independent of the strength of the magnetic field $\left(\chi^{2}=0.9\right.$ for the entire data set) which is $3.7 \%$ below the ideal $\sqrt{2}$. This can be due either to molecules present in the target even with the recombiner valve closed $\left(\alpha_{\text {closed }}=\right.$ $0.879 \pm 0.010, \alpha_{\text {open }}=0$ ), or to incomplete recombination inside the copper recombiner when the recombiner valve is open $\left(\alpha_{\text {open }}=0.089 \pm 0.007, \alpha_{\text {closed }}=1\right)$, or to some combination of the two.

The data analysis was carried out for both extreme assumptions about $\alpha$. The values of $R$ are shown in Figs. 2 and 3, where the error bars are probable errors that include the uncertainty from statistics as well as the systematic uncertainty from the incomplete knowledge of $\alpha_{\text {open }}$ and $\alpha_{\text {closed }}$ discussed above.

The data analysis took into account corrections for contributions from background (2\%) and loss of counts from 


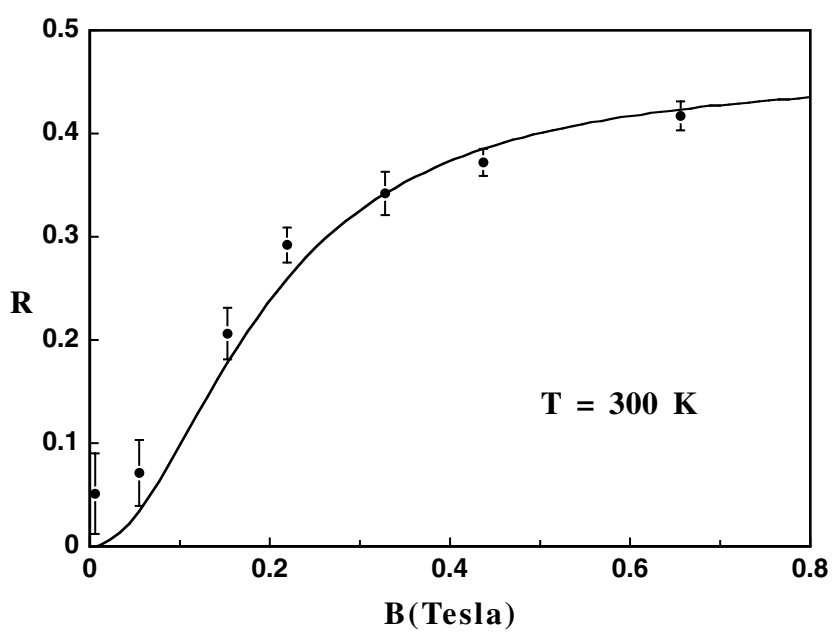

FIG. 2. Fraction $R$ of proton polarization of the $\mathrm{H}$ atoms that is retained in the $\mathrm{H}_{2}$ molecules after recombination on a room temperature copper surface in a magnetic field $B$. The error bars are probable errors that include the uncertainty from statistics as well as the systematic uncertainty from the incomplete knowledge of $\alpha_{\text {open }}$ and $\alpha_{\text {closed }}$ (see text). The curve is calculated from Eq. (4).

dead time $(<2 \%)$. Measurements with $\mathrm{H}_{2}$ in the target cell showed that the detector yield decreased by $(2.1 \pm 0.2) \%$ when the recombiner valve was opened because of a leak in the recombiner. A corresponding correction was applied to the open/closed yield ratios. The fraction $R$ of polarization retained in the molecules shows a strong dependence on magnetic field $B$ applied to the recombiner and target cell (Fig. 2), but almost no dependence on temperature as long as the recombiner temperature is above $70 \mathrm{~K}$ (Fig. 3). As the magnetic field is varied from $5 \mathrm{mT}$ to $0.66 \mathrm{~T}$, the

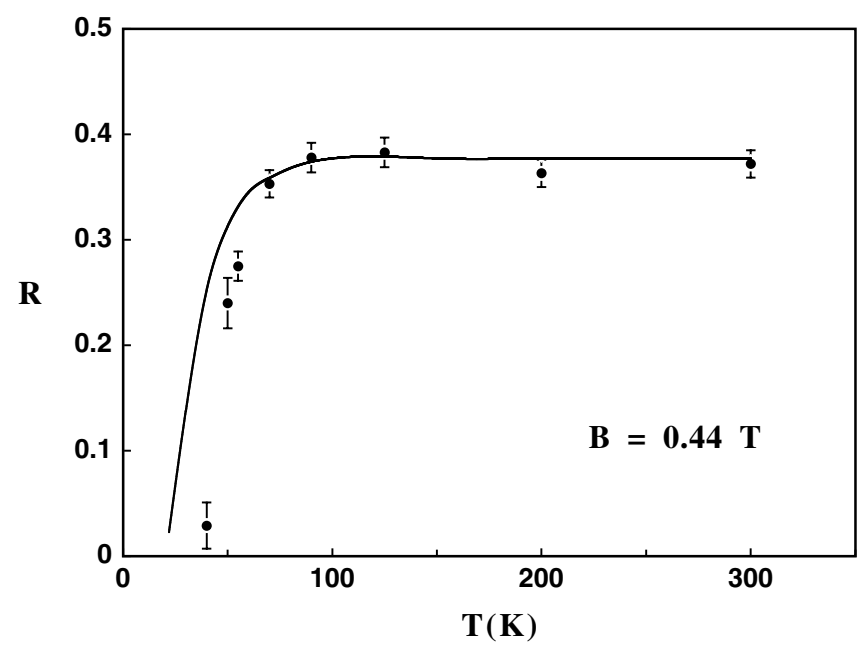

FIG. 3. Fraction $R$ of proton polarization of the $\mathrm{H}$ atoms that is retained in the $\mathrm{H}_{2}$ molecules after recombination on a copper surface at different temperatures $T$ for a fixed magnetic field strength $B=0.44 \mathrm{~T}$. The curve is based on measurements of the polarization of hydrogen atoms in a $\mathrm{Cu}$ cell in which about $65 \%$ of the atoms recombined; see Ref. [15]. nuclear polarization retained by the molecules rises from near zero to $R=0.42 \pm 0.02$.

If no loss in proton polarization occurred, one would observe $R=1$. Assume for a moment that, in a sufficiently strong external magnetic field, the proton spins of the $\mathrm{H}_{2}$ molecules and of the $\mathrm{H}$ atoms in the gas phase are preserved. However, recombination $\mathrm{H}+\mathrm{H} \rightarrow \mathrm{H}_{2}$ requires at least one of the atoms to be adsorbed on the wall prior to recombination, since, at our very small gas densities, volume recombination is completely negligible. The wall atoms are likely to be depolarized by fluctuating fields on the surface, so that the resulting net nuclear polarization of the molecules coming off the wall is half of the original proton polarization of the $\mathrm{H}$ atoms, i.e., $R=1 / 2$. Additional loss of polarization from other processes may arise, e.g., when two atoms on the surface recombine with each other. Indeed, Fig. 2 is not inconsistent with $R$ rising asymptotically to $R \sim 1 / 2$ for large $B$.

We interpret the decrease in $R$ at low $B$ as arising from spin relaxation of the ortho- $\mathrm{H}_{2}$ molecules during the time periods between wall collisions. In free flight, internal molecular fields $B_{c}$ from the spin-rotation interaction $\left(B^{\prime}=2.7 \mathrm{mT}\right)$ and the direct dipole-dipole interaction $\left(B^{\prime \prime}=3.4 \mathrm{mT}\right)$ [12] cause the nuclei to rapidly precess about a direction that is skew to the external field by $\theta=B_{c} / B$. The orientation of $B_{c}$ is randomized by each wall collision. Between successive wall collisions, the component of the polarization along the external field decreases by an amount $B_{c} / B^{2}$; thus, after $n$ wall bounces,

$$
R=R_{0} \exp \left[-n\left(B_{c} / B\right)^{2}\right] \text {. }
$$

We extend the analysis of [12] to obtain $B_{c}=$ $1.19\left[\frac{4}{3} B^{12}+\frac{24}{25} B^{1 / 2}\right]^{1 / 2}=5.4 \mathrm{mT}$ for the $J=1$ rotational state [13]. Accounting for the $12 \%$ of the orthomolecules in the $J=3$ state at room temperature increases $B_{c}$ to $6.1 \mathrm{mT}$. Assuming $R_{0}=0.46$, and an exponential distribution of $n$ with a mean of 1000, gives the curve in Fig. 2. Thus, the molecular polarization is in quantitative agreement with theoretical expectations.

The present calculation of the mean $n$, estimated to be accurate to $\pm 30 \%$, is complicated by the fact that the walls of the recombiner were lined with copper mesh to increase $n$ compared to a bare wall. The calculation of $n$ must consider wall collisions in the target tube, wall collisions before recombination occurs, and account for the $\sim 65 \%$ chance that atoms in the tube diffuse back into the recombiner. A complete Monte Carlo calculation of the distribution of $n$ is in progress.

Thus far, we have tacitly assumed that depolarization of the $\mathrm{H}$ atoms in the gas phase is negligible. Indeed measurements show that those $\mathrm{H}$ atoms that escape recombination retain their polarization even after hundreds of wall collisions. Price and Haeberli $[14,15]$ injected polarized atoms into target cells and ionized the target gas by an electron beam, with subsequent mass analysis of the extracted ions. Depending on wall material and wall temperature, the 
fraction of $\mathrm{H}$ that remained in the cell changed, but their polarization was retained. Only when the wall temperature was lowered below $70 \mathrm{~K}$ did the polarization of the atoms drop very rapidly. This observation is supported by results in the HERMES experiment: when the surface of the target cell was accidentally damaged by the circulating electron beam, about $80 \%$ of the atoms experienced recombination, but the remaining $20 \%$ of the atoms were fully polarized [16].

From the information given above, we can predict how $R$ should depend on wall temperature $T$ of the recombiner. Figure 3 shows data for a fixed magnetic field $B=0.44 \mathrm{~T}$. The only temperature dependence in Eq. (4) arises from the decrease in the $J=3$ population, causing a change in $B_{c}$ from $6.1 \mathrm{mT}$ at $300 \mathrm{~K}$ to $5.4 \mathrm{mT}$ at $40 \mathrm{~K}$. The corresponding change in $R$ at $B=0.44 \mathrm{~T}$ is $3.1 \%$, which is below the accuracy of our data. Thus $R \sim$ const, in agreement with observation down to $T=100 \mathrm{~K}$ (Fig. 3). The rapid drop of $R$ below $70 \mathrm{~K}$ is almost certainly associated with the depolarization of the $\mathrm{H}$ atoms mentioned in the preceding paragraph. In Fig. 3 the curve shows measurements of the polarization of $\mathrm{H}$ atoms in a $\mathrm{Cu}$ cell with 80 wall collisions as a function of wall temperature. The larger number of wall collisions in the present experiment explains why the drop in $R$ occurs at a somewhat higher temperature. Note that, in the present measurements, $T_{\text {cell }}$ was held above $130 \mathrm{~K}$ so that depolarization in the measurement of $P_{\text {at }}$ is negligible.

In summary, measurements of high statistical accuracy have been made of nuclear polarization of $\mathrm{H}_{2}$ molecules which result from the recombination of polarized $\mathrm{H}$ atoms on $\mathrm{Cu}$. It is found that, for the strongest magnetic fields available $(0.66 \mathrm{~T})$, the nuclear polarization of the molecules is nearly half of the polarization of the recombining atoms, which we interpret to result from the recombination of a depolarized atom on the wall and a polarized atom in the gas phase. The decrease of the polarization at lower $B$ is consistent with the assumption that the polarization of the molecules is lost by coupling of the proton spin to the magnetic field of the molecule's rotational motion. The decrease of molecular polarization below $100 \mathrm{~K}$ is expected from available data on the polarization loss of hydrogen atoms in collisions with cold walls.

Further studies are needed of the depolarization mechanism in recombination of $H$ atoms. It would be interesting not only to vary the number of wall collisions but also the hyperfine state composition of the recombining atoms.
Also, measurements on the recombination of deuterium atoms would be of interest. For deuterium, the orthomolecules at room temperature and below are primarily in the $J=0$ rotational state and thus should show less depolarization at low $B$.

We thank Professor L.W. Anderson, Professor L.D. Knutson, and Professor L.W. Bruch for discussions. We thank Mr. Tom Finnessy for his help in the construction and initial tests of the equipment. This work was supported by the U.S. National Science Foundation under Grants No. PHY-9602872, No. PHY-9722556, and No. PHY-9901529, and by the U.S. Department of Energy under Grant No. DOE-FG02-88ER40438.

*Email address: wise@uwnuc0.physics.wisc.edu

${ }^{\dagger}$ Current address: Institut für Kernphysik, Forschungzeutrum Jülich, 52425 Jülich, Germany.

${ }^{\ddagger}$ Current address: Bose Institute, Calcutta, India.

[1] W. Haeberli, in Free and Stored Atomic Beams as Internal Polarized Targets, AIP Conf. Proc. No. 128 (AIP, New York, 1984), p. 251.

[2] A. Airapetian et al., Phys. Lett. B 442, 484 (1998).

[3] F. Rathmann et al., Phys. Rev. C 58, 658 (1998); W. Haeberli et al., Phys. Rev. C 55, 597 (1997).

[4] R. Gilman et al., Phys. Rev. Lett. 65, 1733 (1990).

[5] M. Ferro-Luzzi et al., Phys. Rev. Lett. 77, 2630 (1996).

[6] J. F. J. van den Brand et al., Phys. Rev. Lett. 78, 1235 (1997).

[7] T. Wise, A. D. Roberts, and W. Haeberli, Nucl. Instrum. Methods Phys. Res., Sect. A 336, 410 (1993).

[8] W. Haeberli, Annu. Rev. Nucl. Sci. 16, 373 (1967).

[9] J. S. Price and W. Haeberli, Nucl. Instrum. Methods Phys. Res., Sect. A 349, 321 (1994).

[10] K. Zapfe et al., Rev. Sci. Instrum. 66, 28 (1995).

[11] B. Lorentz et al., Phys. Rev. C 61, 54002 (2000).

[12] A. Abragam, Principles of Nuclear Magnetism (Oxford University Press, New York, 1961), p. 317.

[13] The factor 1.19 accounts for the reduced coupling of the nuclei to the external field due to the rotational magnetic moment.

[14] J. S. Price and W. Haeberli, Nucl. Instrum. Methods Phys. Res., Sect. A 326, 416 (1993).

[15] J. S. Price, Ph.D. thesis, University of Wisconsin, 1993. [available as dissertation No. 9318638, from Dissertation Express (http://wwwlib.umi.com/dxweb/)]. For the curve in Fig. 3, the results were normalized by a factor of 0.46 .

[16] HERMES Collaboration, T. Wise (private communication). 